\title{
Prevalencia De Parasitismo Intestinal Y Su Asociacion Con El Estado Nutricional En Niños Del Municipio De Galapa, Atlántico-Colombia
}

\author{
Karen Esther Muñoz, Salas \\ Magister en Ciencias Básicas Biomédica, Universidad del Norte \\ Alma del Socorro Polo Barrios \\ Magister en Biología, Universidad Estatal Moscú. \\ Docente. Dpto. de química y biología, Universidad del Norte
}

Carmiña Lucia Vargas Zapata

Doctorado en Bioquímica, Universidad de California Santa Bárbara

Docente Dpto. de biología, Universidad del Atlántico

\section{María del Pilar Garavito Galofre}

Doctorado en neurociencias y Genética Molecular, Rutgers University

Docente. Dpto. de Medicina, Universidad del Norte

\section{Carolina Maestre Gonzales}

Pediatra del Hospital General del Norte, Universidad del Norte

\section{Juan David Rodríguez Macías}

Magister en Ciencias Básicas Biomédica. Universidad del Norte

Docente. Dpto. de química y biología de la Universidad del Norte

Docente. Dpto. de Ingeniería Ambiental de la Universidad de la Costa

Doi: 10.19044/esj.2018.v14n36p12 URL:http://dx.doi.org/10.19044/esj.2018.v14n36p12

\begin{abstract}
The purpose of this research is to characterize the prevalence of intestinal parasites in children between two to ten years old, and its relation with their nutritional status. 123 children were assessed, from a secondary school in the town of Galapa at the Atlantico department, in the Colombian Caribbean region (Colombia). After informed consent from their parents, after children were weighed and carved, stool samples were collected in triplicate/duplicate. Samples were processed using the sedimentation technique by centrifugation and kato katz. Our results showed parasitism in $39 \%$ of the population with a $38 \%$ of the individuals infected by protozoa and a $0.8 \%$ with helminths. Analysis by gender showed that there were not statistical significant difference with $20.3 \%$ boys were infected compared with $17.1 \%$ of the girls. Furthermore, parasites prevalence was not statistically different when analyzed by gender. The parasites with the highest prevalence
\end{abstract}


were: Giardia duodenalis $(7.7 \%)$, Blastocystis spp $(6.8 \%)$ and Endolimax nana $(3.0 \%)$ and children were found infected with polyparasitism by $G$. duodenalis + Blastocystis spp $(1.7 \%)$ y Entamoeba histolytica + Endolimax nana $(0.4 \%)$. The children that presented parasitism had a normal size and average weight of $124.8 \mathrm{~cm} 27.5 \mathrm{~g}$ respectively. Most children with parasitism had a normal nutritional status $(21.1 \%)$, some were overweight $(8.1 \%)$ and obesity $(4.9 \%)$, and there were no children at risk of thinness and malnutrition. Our findings suggest that there is not significant correlation between the presence of parasites and the dietary habits in this population.

Keywords: Prevalence, Intestinal Parasites, Nutrition, Children, Atlantic

\section{Resumen}

El objetivo del estudio fue determinar la prevalencia de parasitismo intestinal en una población infantil en relación al estado nutricional de los infantes con edades entre los 2 y 10 años. Para ello se seleccionaron 123 infantes de instituciones educativas del municipio de Galapa-Atlántico (Colombia). Dichos infantes fueron pesados y tallados y donaron muestras de heces por duplicado/triplicado, las cuales fueron procesadas mediante la técnica de sedimentación por centrifugación y kato katz. En total la población parasitada fue del 39\% de los cuales el $38.2 \%$ correspondió a protozoos y el $0.8 \%$ a helmintos. Al discriminar entre niños y niñas que presentaron parásitos, se encontró un $20.3 \%$ y $17.1 \%$ respectivamente y no se encontró diferencias estadísticamente significativas entre sexos ni en relación a la especie de parásitos presentes. Los parásitos con mayor prevalencia fueron: Giardia duodenalis (7.7 \%) y Blastocystis spp (6.8\%), seguido de Endolimax nana (3.0 \%). Los menos frecuentes fueron: Entamoeba coli (0.9\%), Entamoeba histolytica $(0.4 \%)$ y Hymenolepis nana $(0.4 \%)$. Además, se encontró infantes infectados con poliparasitismo, de los cuales corresponden a los parásitos G. duodenalis + Blastocystis spp (1.7\%) y Entamoeba histolytica + Endolimax nana $(0.4 \%)$. Los infantes parasitados, tuvieron una talla y peso promedio de $124.8 \mathrm{~cm}$ y $27.5 \mathrm{~g}$ respectivamente. La mayoría de los infantes con parasitismo presentaron un estado nutricional normal $(21.1 \%)$, algunos tenían sobrepeso $(8.1 \%)$ u obesidad $(4.9 \%)$ y no se encontraron infantes con riesgo de delgadez y desnutrición. No se observó asociación estadísticamente significativa entre la presencia de parásitos y el estado nutricional en la población infantil.

Palabras claves: Prevalencia, parasitismo intestinal, nutrición, niños, Atlántico 


\section{Introduction}

El parasitismo intestinal representa un problema de salud pública y la población infantil es la más afecta por la parasitosis, la mayoría de los niños se encuentran en etapa exploratoria y su sistema inmunológico se encuentra poco desarrollado, por lo que son propensos a contraer parásitos (Zonta et al. 2007). Villalba-Vizcaíno et al. 2015, en Santa Marta, Magdalena, encontraron que el $72.7 \%$ de los niños presentaban parásitos intestinales. Garzón et al. 2015, en Caquetá determinaron estudiaron los factores de riesgos en niños con parásitos de 5 años residentes de la Candelaria; el estudio mostró una prevalencia del $90 \%$ de niños infectados por parásitos, los trabajos reflejan que el parasitismo sigue siendo un problema en la actualidad y es importante tomar medidas de control y prevención.

Otra variable adicional que afecta a la población infantil, es mantener un estado nutricional normal, debido a que los niños mantienen dietas inadecuadas (cantidades requeridas) y consumen alimentos en forma desbalanceada y poco saludables. Por ende, encontramos niños con desnutrición, sobrepesos y obesidad (Chávez, 2013; Morr, 2013).

Se han realizado diferentes estudios sobre parasitismo en los cuales se menciona que pueden afectar el estado nutricional del niño (Rodríguez et al. 2016; Solano et al. 2008). Claros (2015), relacionó el estado nutricional de los niños infectados con la parasitosis intestinal en Cartagena y encontró que el $58.2 \%$ de los infantes poseían un peso adecuado para la talla, el 15.7\% de los niños estaban en riesgo de bajo peso para la talla o en su defecto con sobrepeso; el $5.4 \%$ con desnutrición y el $4.7 \%$ con obesidad. Rodríguez et al. (2014), encontraron prevalencia de parásitos intestinales en el $65.3 \%$ de la población infantil del municipio de Soracá-Boyacá, (Colombia) y de acuerdo al estado nutricional encontraron que el $30 \%$ estaba en riesgo de retraso en el crecimiento, el $22 \%$ ya poseía retraso en el crecimiento, el $3 \%$ presentaba sobrepeso u obesidad y el 35\% se encontraba con riesgo de desnutrición.

En Colombia, la encuesta nacional de parasitismo 2012-2014, informo que $81 \%$ de los niños presentaba infecciones por parásitos intestinales, de los cuales el 29.62\% eran por geohelmintos (Ministerio de Protección Sociales et al. 2015); estudios recientes reportan disminución en las infecciones por parásitos en niños en algunos partes de Colombia. Cardona-Arias \& BedoyaUrrego, (2012), reportan $74.1 \%$ en comunidades marginales de Medellín (Colombia). Londoño et al. (2008) reportan un 54.7\% en una zona urbana en Calarcá (Colombia), Rodríguez et al. (2016) en Izya Boyacá un 50\% (Cardona-Arias \& Bedoya-Urrego, 2012; Londoño et al. 2008; Rodríguez et al. 2016).

Las pocas investigaciones que se han reportado en el departamento del Atlántico son en los municipios de Galapa, Santo Tomás y el corregimiento de la playa encontrando en el 2010 una mayor prevalencia de parásitos 
intestinales en Santo Tomas de $82 \%$, seguido de Galapa con $72 \%$ (Londoño et al. 2010). En el 2015, se reportó en el municipio de Galapa y el corregimiento de la Playa un 50\% y 56.8\% de parásitos (Fillot et al. 2015). Se observó una alta prevalencia del parásito reportado en las investigaciones realizadas en los diferentes municipios del departamento Atlántico, pero estos estudios se realizaron desde 2010 a 2015. Por ende, se desconoce la prevalencia actual de parásitos intestinales en el departamento del Atlántico. Es por ello que el presente estudio se desarrolló el municipio Galapa, Atlántico (Colombia). Los objetivos fueron: determinar la frecuencia de parasitismo intestinal y el estado nutricional de los niños e identificar posible asociación entre ambos.

\section{Metodología}

\section{Área y sujetos de estudios}

La presente investigación es de tipo descriptiva de corte trasversal, y se llevó a cabo en Colombia en el municipio de Galapa-Atlántico en la institución educativa Francisco de Paula Santander. Para conocer la infección por parásitos intestinales se estudió 123 niños de en edades comprendidas entre 2 -10 años.

El proyecto de investigación y el procedimiento de la colecta de muestra fue socializada con los padres de familia, con el fin de dar a conocer su importancia y obtener su aceptación en la participación de la investigación. Cada padre de familia firmó un consentimiento informado y los infantes un asentimiento, A cada, padre de familia se le entregaron tres frascos colectores de heces y se estableció un cronograma para la colecta según el nivel educativo (Desde párvulo hasta quinto de primaria). Una vez obtenidas las muestras de heces se trasladaron al laboratorio multifuncional de la Universidad del Norte, donde las muestras se analizaron por la técnica de sedimentación por centrifugación y kato katz descrita por Berenguer, 2007.

\section{Antropometría}

Para evaluar el estado nutricional, se diseñaron planillas donde se registró información correspondiente al peso y talla de los niños cuyas edades oscilaban entre los 2-10 años y solo a los niños menores de 5 años se le registró el perímetro cefálico.

La toma de las medidas antropométricas se realizó de la siguiente manera:

Peso: Se utilizó una báscula de piso, ubicada sobre una superficie plana, horizontal y firme, previamente calibrada. Los niños debían estar sin zapatos, en posición supina, con los brazos relajados a los costados y la mirada al frente, luego se procedió a realizar las lecturas correspondientes de cada niño. 
Talla: Se colocó un metro ubicado a $50 \mathrm{~cm}$ de la base del piso y con una escuadra se hizo la lectura.

Perímetro cefálico: Se levantó la cabeza del niño con una mano y se deslizó una cinta métrica por debajo de ella, luego se colocó la cinta al nivel del occipital y parte media de la frente y se realizó lectura.

El estado nutricional para los niños menores de 5 años se realizó a través de la relación peso /talla del puntaje Z-Score. El estado nutricional de los niños mayores de 5 años, se realizó a través de la relación índice de masa corporal (IMS)/ edad, luego el resultado obtenido se comparó con el puntaje Z-Score de referencia de WHO, 2006 para niños menores de 5 años de edad y para niñas, niños y adolescentes de 5 a 18 años de edad (WHO, 2006; De Oneis \& Blossner, 2003).

\section{Análisis estadístico}

El procesamiento y análisis de datos se realizó mediante los programas Office Excel versión 9, SPSS® Statistics versión 22.0 (IBM Corp, EE. UU), Epidat, versión 3.1, WHO Anthro versión 3.3 y WHO Anthro plus versión 1.04. En primera instancia se tabularon los datos de peso y talla en WHO Anthro versión 3.3 y WHO Anthro plus versión 1.04, luego se clasifico el estado nutricional. En el programa Office Excel se tabulo el diagnóstico parasitario y el estado nutricional. Los datos obtenidos en el programa Excel, se llevaron al SPSS® Statistics donde se determinó la distribución de los datos por el test de Kolmogorov Smirv y se aplicó la prueba de Levene para determinar la homocedasticidad de las varianzas

Se aplicó estadística descriptiva calculando medidas de tendencia central (promedio, rango, mínimos y máximos), número y porcentajes según la variable evaluada. Se calculó el chí cuadrados $\left(\mathrm{X}^{2}\right)$ y el valor de $\mathrm{P}$ a cada variable.

Se estableció la asociación entre el parasitismo y el estado nutricional, con la razón probabilidad (OR), intervalos de confianza (95\%) y el valor de $\mathrm{P}$, calculados Epidat, versión 3.1.

\section{Resultados}

La edad media de los infantes estudiados, fue $7.87 \pm 1.7$ con rango de 6 años. De los cuales $68(54.5 \%)$ eran niñas y $55(44.7 \%)$ eran niños. De la población total que se estudió en Galapa -Atlántico, el $39.0 \%$ presentaba algún tipo de parásitos (Tabla 1).

Tabla 1. Parasitismo intestinal en una población infantil menor de 10 años que asisten a una institución educativa de Galapa, Atlántico.

\begin{tabular}{llll}
\hline \multirow{3}{*}{ Parásitos } & & $\mathrm{n}$ & $\%$ \\
& $\mathrm{Si}$ & 48 & 39.0 \\
& $\mathrm{No}$ & 75 & 60.9 \\
\hline
\end{tabular}


La población infantil de las niñas y niños estaban infectados por parásitos en un $20.3 \%$ y $17.1 \%$ respectivamente, y no se encontraron diferencias estadísticamente significativas entre sexo y las especies de parásitos presentes $\left(\mathrm{X}^{2}=0.02 ; \mathrm{P}=0.87\right.$ ) (Tabla 2).

Tabla 2. Parasitismo intestinal por sexo en infantes menor de 10 años que asisten a una institución educativa de Galapa, Atlántico.

\begin{tabular}{lllll}
\hline & \multicolumn{3}{c}{ Parásitos } \\
\hline & & Si & & No \\
Sexo & $\mathrm{n}$ & $\%$ & $\mathrm{~N}$ & $\%$ \\
Niñas & 25 & 20.3 & 43 & 35.0 \\
Niños & 21 & 17.1 & 34 & 27.6 \\
\hline
\end{tabular}

*Indica diferencias estadísticamente significativas. $\mathrm{X}^{2}=0.02 ; \mathrm{P}=0.87$

Se encontró que el $39.0 \%$ de la población estaba infectada con parásitos, y el $38.2 \%$ correspondió a protozoos y el $0.8 \%$ a helmintos (Tabla 3).

Tabla 3. Prevalencia general de parásitos intestinales en una población de niños menor de

\begin{tabular}{ccl}
10 años que asisten a una institución educativas de Galapa, Atlántico. \\
\hline Prevalencia & $\mathbf{N}$ & $\mathbf{n \%}$ \\
\hline General & 48 & 39.0 \\
Protozoos & 47 & 38.2 \\
Helmintos & 1 & 0.8 \\
Negativas & 75 & 60.9 \\
\hline
\end{tabular}

Los parásitos con mayor prevalencia fueron: Giardia duodenalis $(7.7$ $\%)$ y Blastocystis spp (6.8\%), seguido de Endolimax nana (3.0\%). Los menos frecuentes fueron: Entamoeba coli $(0.9 \%)$, Entamoeba histolytica $(0.4 \%)$ y Hymenolepis nana $(0.4 \%)$. Además, se encontró infantes infectados con poliparasitismo, de los cuales corresponden a los parásitos G. duodenalis + Blastocystis spp (1.7\%) y Entamoeba histolytica + Endolimax nana $(0.4 \%)$ (Tabla 4).

Tabla 4. Prevalencia por especies de parásitos intestinales en niños menor de 10 años que asisten a una institución educativas de Galapa, Atlántico.

\begin{tabular}{|c|c|c|c|}
\hline Prevalencia por especies & & $\mathbf{n}$ & $\%$ \\
\hline \multirow{4}{*}{ Parasitismo por una especie } & Giardia duodenalis & 18 & 7.7 \\
\hline & Blastocystis spp & 14 & 6.8 \\
\hline & Endolimax nana & 7 & 3.0 \\
\hline & Entamoeba coli & 2 & 0.9 \\
\hline \multirow{6}{*}{ Parasitismo por dos especies } & Entamoeba histolytica & 1 & 0.4 \\
\hline & Hymenolepis nana & 1 & 0.4 \\
\hline & G. duodenalis + Blastocystis spp & 4 & 1.7 \\
\hline & G. duodenalis + Endolimax nana & 1 & 0.4 \\
\hline & Negativas & 75 & 60.9 \\
\hline & Total $(\mathrm{N})$ & 123 & \\
\hline
\end{tabular}

n: Cantidad de niños que presentaron parásitos; $n \%$ : Porcentaje de niños con parásitos de la población total. 
Los indicadores antropométricos de los niños menores de 5 años se encontraron dentro de la normalidad (10.6\%), en los casos de los niños mayores de 5 años el $50.4 \%$, se encontró dentro de la normalidad el $20.3 \%$ tenía sobrepeso y el $15.4 \%$ presentaba obesidad. No se encontraron niños con riesgo de delgadez y delgadez (Tabla 5).

Tabla 5. Valoración nutricional en niños menores de 10 años que asisten a una institución educativas de Galapa, Atlántico.

\begin{tabular}{|c|c|c|c|c|c|}
\hline $\begin{array}{l}\text { Menores de } 5 \\
\text { años }\end{array}$ & Normal & $\begin{array}{l}\text { Riesgo } \\
\text { Desnutrición }\end{array}$ & $\begin{array}{l}\text { Desnutrición } \\
\text { Aguda }\end{array}$ & Sobrepeso & Obesidad \\
\hline Peso/Talla & $13(10.6 \%)$ & $0 \%$ & $0 \%$ & $3(2.4 \%)$ & $1(0.8 \%)$ \\
\hline $\begin{array}{l}\text { Mayores de } 5 \\
\text { años }\end{array}$ & Normal & Riesgo Delgadez & Delgadez & Sobrepeso & Obesidad \\
\hline IMC/Edad & $62(50.4 \%)$ & $0 \%$ & $0 \%$ & $25(20.3 \%)$ & $19(15.4 \%)$ \\
\hline
\end{tabular}

Los niños que presentaron parasitismo intestinal, tuvieron una talla y peso promedio de $124.8 \mathrm{~cm}$ y $27.5 \mathrm{~g}$ respectivamente. La mayoría de los niños presentaron un estado nutricional normal, $(21.1 \%)$, algunos tenían sobrepeso $(8.1 \%)$ u obesidad (4.9\%). No se presentaron niños con riesgo de delgadez y desnutrición. No se encontró asociación estadísticamente significativa entre la presencia de parásitos y el estado nutricional en la población infantil en ninguno de los casos estudiados (Tabla 6).

Tabla 6. Estado nutricional asociado al parasitismo en niños menores de 10 años que asisten a una institución educativas de Galapa, Atlántico.

\begin{tabular}{|c|c|c|c|c|c|c|}
\hline \multirow{3}{*}{$\begin{array}{c}\text { Estado } \\
\text { nutricional }\end{array}$} & \multicolumn{4}{|c|}{ Parásitos } & \multirow{2}{*}{\multicolumn{2}{|c|}{ Parámetros estadísticos }} \\
\hline & & & & & & \\
\hline & $\mathbf{n}$ & $\%$ & $\mathbf{n}$ & $\%$ & Valor de $\mathbf{P}$ & $95 \%)$ \\
\hline Normal & 26 & 21.1 & 49 & 39.8 & 0.87 & $\begin{array}{c}1.06 \\
(0.78-1.43)\end{array}$ \\
\hline Sobrepeso & 10 & 8.1 & 18 & 14.6 & 0.84 & $\begin{array}{c}1.09 \\
(0.73-1.62)\end{array}$ \\
\hline Obesidad & 6 & 4.9 & 14 & 11.4 & 0.66 & $\begin{array}{c}0.79 \\
(0.46-1.38)\end{array}$ \\
\hline
\end{tabular}

n: Cantidad de niños con parásitos y sin parásitos con su estado nutricional, OR (IC al 95\%): Razón de probabilidades con intervalos de confianza al 95\%; * diferencias estadísticamente significativas con $\mathrm{P} \leq 0.05$.

\section{Discusion}

Se determinó una baja prevalencia general de parasitosis intestinales en la población infantil de Galapa, lo cual coincide con los resultados alcanzados por De la Barquera \& Miramontes-Zapata (2010) en México; sin embargo, nuestros resultados difieren del estudio de Miranda, (2014) hecho en Venezuela y Agudelo-Lopez et al. (2008) realizado en la costa Atlántica Colombiana quienes observaron prevalencias superiores $(63.1 \%$ y $90 \%$ respectivamente). 
Al discriminar por sexo, se observó que no hay diferencias estadísticamente significativas entre el porcentaje de niños infectados por sexos ni en las especies de parásitos presentes, similar a lo que sucedió en los estudios de Al Rumhein et al. (2005). Devera et al. (2007) y Barra et al. (2016). Esto podría deberse a que comparten actividades similares y a las similares características sociodemográficas y sanitarias de la zona de estudio.

Se encontró una alta prevalencia por protozoos en el municipio de Galapa, el protozoo más predomínate fue Giardia duodenalis y Blastocytis spp similar a lo encontrado en las investigaciones de Tabares et al. 2008 en Antioquia (Colombia), Tedesco et al. (2012) en Venezuela, De la OssaMerlano et al. 2007 en Barranquilla y tres municipios de Atlántico (Campo de la Cruz, Piojo y Soledad), lo cual podría deberse a los factores ambientales (como clima y suelo) y al consumo de alimentos y bebidas contaminadas, especialmente lechuga y agua sin hervir. Barahona-Rondon et al. 2003, estudio la blastocistosis en personas con edades entre los 5 a 80 años, encontró una alta prevalencia por Blastocytis spp (51.4\%) y que se asociaba significativamente con el consumo del agua sin hervir, lo cual pudo haber sucedió en nuestro estudio (Soriano et al. 2005; Devera et al. 2006; EspinosaMuñoz et al. 2015). Otro factor que puede influir en la alta carga de protozoos son el nivel de escolaridad de la madre y los hábitos higiénicos como lavado de mano después de comer y defecar (Rodríguez et al. 2011).

No se encontró asociación entre el estado nutricional y el parasitismo intestinal en los niños estudiados, al igual que lo observado en la zona sur de la Ciudad de Valencia, Venezuela (Solano et al. 2008) y en Huánuco Perú (Moreano et al. 2010), quienes no encontraron asociación significativa entre nivel de desnutrición y parasitismo. Los autores lo atribuyen al estado basal nutricional antes de adquirir la infección parasitaria, a los grados de infección parasitaria y al patrón de ingesta dietética durante la infección como lo menciona Moreno et al. (2010).

En los estudio realizados por Mendoza et al. (2009) en Cuba, Ordoñez \& Angulo, (2002) en el Amazonía Colombiano, Quihui-Cota et al. (2004) en México determinaron que la infección por enteroparásitos se encontraban relacionadas con la pobreza, la edad y el estado nutricional de niños y adolescentes.

\section{Conclusion}

Se presentó una baja prevalencia de parásitos intestinales en el municipio de Galapa (Atlántico) y la mayoría de la población infantil presentó un estado nutricional normal. No se encontró asociación entre la presencia de parásitos y el estado nutricional de los niños

El estudio permitió obtener información acerca del problema de parasitismo intestinal en el municipio de Galapa (Atlántico), con el fin de que 
los padres de familia tomen medidas de prevención y control en cuanto a la presencia de parásitos detectados en los niños.

\section{Agradecimientos}

Al Departamento del Atlántico y a COLCIENCIAS bajo la Convocatoria No. 673 (2014). A la doctora Celia Trujillo y doctor Carlos Silvera por brindar su apoyo en la logística y valoración antropométrica de los niños.

\section{References:}

1. Agudelo-Lopez, S., Gómez-Rodríguez, L., Coronado, X., Orozco, A., Valencia-Gutiérrez, C., Restrepo-Betancur, L. F., ... \& Botero-Palacio, L. (2008). Prevalencia de parasitosis intestinales y factores asociados en un corregimiento de la costa atlántica colombiana. Revista de salud pública, 10(4), 633-642.

2. Al Rumhein, F., Sánchez, J., Requena, I., Blanco, Y., \& Devera, R. (2005). Parasitosis intestinales en escolares: relación entre su prevalencia en heces $\mathrm{y}$ en el lecho subungueal. Revista Biomédica, 16(4), 227-238.

3. Barahona-Rondón, L., Maguiña-Vargas, C., Náquira-Velarde, C., Terashima, I., \& Tello, R. (2003). Blastocystosis humana: Estudio prospectivo, sintomatología y factores epidemiológicos asociados. Revista de Gastroenterología del Perú, 23(1), 29-35

4. Barra, M., Bustos, L., \& Ossa, X. (2016). Desigualdad en la prevalencia de parasitosis intestinal en escolares de una escuela urbana y dos rurales de la comuna de Puerto Montt. Revista médica de Chile, 144(7), 886-893.

5. Berenguer, J. G. (2007). Manual de parasitología: morfología y biología de los parásitos de interés sanitario (Vol. 31). Edicions Universitat Barcelona.

6. Cardona-Arias, J., \& Bedoya-Urrego, K. (2013). Frecuencia de parásitos intestinales y evaluación de métodos para su diagnóstico en una comunidad marginal de Medellín, Colombia. Iatreia, 26(3), 257268.

7. Chávez, O., \& Díaz, S. (2013). Consumo de comida rápida y obesidad, el poder de la buena alimentación en la salud. Revista Iberoamericana para la Investigación y el Desarrollo Educativo: RIDE, 4(7), 176-199.

8. Claros, B. P. (2015). Parasitosis intestinal relacionada con el estado nutricional de los niños de 2 a 5 años en hogares comunitarios del Instituto Colombiano de Bienestar Familiar (ICBF) de la ciudad de Cartagena de Indias. 
9. De la Barquera, M., \& Miramontes-Zapata, M. (2011). Parasitosis intestinales en 14 comunidades rurales del altiplano de México. Revista Latinoamericana de Patología Clínica y Medicina de Laboratorio, 58(1), 16-25.

10. De la Ossa-Merlano, N., Falconar, A., Llinás-Solano, H., \& RomeroVivas, C. (2007). Manifestaciones clínicas y factores de riesgo asociados a la infección por Cryptosporidium en pacientes de Barranquilla y tres municipios del Atlántico (Colombia). Salud Uninorte, 23(1). 19-31.

11. De Onis, M., \& Blössner, M. (2003). The World Health Organization global database on child growth and malnutrition: methodology and applications. International journal of epidemiology, 32(4), 518-526.

12. Devera, R., Blanco, Y., González, H., \& García, L. (2006). Parásitos intestinales en lechugas comercializadas en mercados populares y supermercados de Ciudad Bolívar, Estado Bolívar, Venezuela. Revista de la Sociedad Venezolana de Microbiología, 26(2), 100-107.

13. Devera, R., Ortega, N., \& Suárez, M. (2007). Parásitos intestinales en la población del Instituto Nacional del Menor, Ciudad Bolívar, Venezuela. Revista de la sociedad venezolana de microbiología, 27(1), 349-363.

14. Espinosa-Muñoz, D., Gómez, N., Polanco, L., Arias, J, \& Osorio, L. (2015). Prevalencia de parasitismo intestinal en la comunidad Seminke del resguardo indígena Wiwa de la Sierra Nevada de Santa Marta, 2014. Archivos de Medicina, 11(26)1-10.

15. Fillot, M., Guzman, J., Cantillo, L., Gómez, L., Sánchez Majana, L., Acosta, B. M., \& Sarmiento-Rubiano, L. (2015). Prevalencia de parásitos intestinales en niños del Área Metropolitana de Barranquilla, Colombia. Revista Cubana de Medicina Tropical, 67(3), 1-13.

16. Garzón, A., Alvarez-Mota, Chicue, J., López, D., \& Mendoza, C. (2015). Parasitosis intestinal y factores de riesgo en niños de los asentamientos subnormales, Florencia- Caquetá, Colombia. Facultad Nacional de Salud Pública, 33(2), 171-180.

17. Londoño-Franco, A., \& Mejía-López, S. (2008). Factores de riesgo para malnutrición relacionados con conocimientos y prácticas de alimentación en preescolares de estrato bajo en Calarcá. 20062007. Revista Gerencia y Políticas de Salud, 7(15),77-90.

18. Londoño, A., Mejía, S., \& Gómez-Marín, J. (2009). Prevalencia y factores de riesgo asociados a parasitismo intestinal en preescolares de zona urbana en Calarcá, Colombia. Revista de Salud Pública, 11 (1), $72-81$.

19. Mendoza, P., Significación, F., \& Angulo-Bazán, Y. (2009). Enteroparasitismo en niños y su relación con la pobreza y estado 
nutricional. Ciencia e Investigación Medico Estudiantil Latinoamericana, 14(1). 49-54.

20. Ministerio de Salud y Protección Social., \& Universidad de Antioquia. (2015). Encuesta nacional de parasitismo intestinal en población escolar Colombia, 2012 - 2014. Medellín-Colombia: Facultad Nacional Salud Pública, Universidad de Antioquia, El Ministerio.

21. Miranda, J. A. (2015). Prevalencia de parasitosis intestinales en unidades educativas de Ciudad Bolívar, Venezuela. Revista Cuidarte, 6(2), 1076-1083.

22. Moreano, B., Gabriel, C., Cahuana, J., Cárdenas, J. K., Ortiz, B., Ruth, N., ... \& Jennifer, E. (2013). Nivel de pobreza y estado nutricional asociados a parasitosis intestinal en estudiantes, Huánuco, Perú, 2010. Anales de la Facultad de Medicina, 74(4), 301-305. doi:http://dx.doi.org/10.15381/anales.v74i4.2702.

23. Morr, M. M. (2013). Frecuencia de obesidad y sobrepeso en una Institución de Salud de la Ciudad de Barranquilla. Ciencia $e$ Innovación en Salud, 1(1), 33-49.

24. Ordóñez, L., \& Angulo, E. (2002). Desnutrición y su relación con parasitismo intestinal en niños de una población de la Amazonia colombiana. Biomédica, 22(4), 486-98.

25. Quihui-Cota, M., Valencia, D., Crompton, S., Phillips, P., Hagan, S., Díaz-Camacho, A. Triana, T. (2004). Prevalence and intensity of intestinal parasitic infections in relation to nutritional status in Mexican schoolchildren. Trans R Soc Trop Med Hyg. 98(11): 653659.

26. Rodríguez, A., Camacho, J., \& Baracaldo, C. (2016). Estado nutricional, parasitismo intestinal y sus factores de riesgo en una población vulnerable del municipio de Iza (Boyacá), Colombia año 2013. Revista chilena de nutrición, 43(1), 45-53.

27. Rodríguez, C., Rivera, M., Cabanillas, Q., Pérez, M., Blanco, H., Gabriel, J., et al. (2001). Prevalence and risk factors for intestinal parasitism in students from Los baños del Inca district, Peru. Scientia, 3(1): 181-186.

28. Rodríguez-Sáenz, A. Y. (2015). Factores de riesgo del parasitismo intestinal en niños escolarizados de una institución educativa del municipio de Soracá-Boyacá, Colombia. Univ. salud, 17(1), 112-120.

29. Solano, L., Acuña, I., Barón, M. A., Morón de Salim, A., \& Sánchez, A. (2008). Influencia de las parasitosis intestinales y otros antecedentes infecciosos sobre el estado nutricional antropométrico de niños en situación de pobreza. Parasitología latinoamericana, 63(1234), 12-19. 
30. Soriano, S., Manacorda, A., Pierangeli, N., Navarro, M., Giayetto, A., Barbieri, L., ... \& Basualdo, J. (2005). Parasitosis intestinales y su relación con factores socioeconómicos y condiciones de hábitat en niños de Neuquén, Patagonia, Argentina. Parasitología latinoamericana, 60(4), 154-161.

31. Tabares, L., \& González, L. (2008). Prevalencia de parasitosis intestinales en niños menores de 12 años, hábitos higiénicos, características de las viviendas y presencia de bacterias en el agua en una vereda de Sabaneta, Antioquia, Colombia. Iatreia, 21(3), 253-259.

32. Tedesco, R., Blanco, Y., \& Devera, R. (2012). Baja frecuencia de geohelmintos en cuatro comunidades rurales del municipio Heres, estado Bolívar, Venezuela. SABER. Revista Multidisciplinaria del Consejo de Investigación de la Universidad de Oriente, 24(2), 151159.

33. Villalba-Vizcaíno, V., Buelvas, Y., Arroyo-Salgado, B., \& Castro, L. (2018). Molecular identification of Giardia intestinalis in two cities of the Colombian Caribbean Coast. Experimental parasitology, 189(1) 17.

34. World Health Organization. (2006). Multicentre Growth Reference Study Group. Assessment of differences in linear growth among populations in the WHO Multicentre Growth Reference Study. Acta Pediatrica, 450(95):56-65.

35. World Health Organization. AnthroPlus for personal computers manual: Software for assessing growth of the world's children and adolescents. Geneva: World Health Organization; 2011. doi: http://www.who.int/childgrowth/software/en/

36. Zonta, M., Navone, G., \& Oyhenart, E. (2007). Parasitosis intestinales en niños de edad preescolar y escolar: situación actual en poblaciones urbanas, periurbanas y rurales en Brandsen, Buenos Aires, Argentina. Parasitología latinoamericana, 62(1-2), 54-60. 